\title{
La Consulta Popular como mecanismo de participación ciudadana para evitar actividades mineras
}

\section{The referendum as a mechanism for citizen participation to prevent mining}

\author{
MIGUEL ÁNGEL GARCÉS VILLAMIL \\ Abogado, Magister en Ciencias del Estado y Doctorante en Ciencia Politica. Docente Investigador del Programa de Derecho \\ de la Universidad Autónoma del Caribe. \\ miguel.garces@uac.edu.co \\ Calle 90 No.46-112 \\ Universidad Autónoma del Caribe \\ Facultad de Jurisprudencia 2do.Piso

\section{WILLIAM GABRIEL RAPALINO BAUTISTA} \\ Abogado, Candidato a Magister en Derecho Internacional de los Derechos Humanos. Profesor de Tiempo Completo del \\ Programa de Derecho de la Universidad Autónoma del Caribe. \\ william.rapalino@uac.edu.co
}

Para citar este artículo

Garcés Villamil, M \& Rapalino Bautista, W (2015). La Consulta Popular como mecanismo de participación ciudadana para evitar actividades mineras. Justicia Juris, 11(1), 52-62.

Recibido: Noviembre 14 de 2014

Aceptado: Febrero 26 de 2015

\begin{abstract}
El importante desarrollo de actividades mineras en Colombia durante los últimos lustros ha ocasionado que se incrementen de manera importante las actividades de exploración y explotación de recursos naturales. Los descubrimientos de importantes yacimientos, como La Colosa en el Departamento del Tolima han traído como consecuencia el inicio de proyectos de gran magnitud que traerán consecuencias en el entorno de las comunidades. La aparición de consultas populares que buscan la opinión de los ciudadanos con la finalidad de impedir la realización de dichas actividades ha provocado una discusión sobre la autonomía de los entes territoriales con respecto a estos temas. En este artículo de reflexión se hace una mención al estado de la actividad minera en Colombia, se revisa el marco legal de las consultas populares, las desarrolladas en temas mineros y la respuesta legal del Gobierno Nacional que busca conservar la autonomía del manejo del ordenamiento minero. Se menciona las decisiones legales adoptadas a la fecha y en las conclusiones se plantea que la manera en que se resuelva la misma dará luces si en realidad el país un Estado Social de Derecho.
\end{abstract}

Palabras Clave: Autonomía Territorial, Consulta Popular, Democracia Participativa, Minería, 


\begin{abstract}
The important development of mining in Colombia during the last periods has caused an increase of activities of exploration and exploitation of natural resources. The discoveries of important deposits, since The Colosa deposit in the department of the Tolima, have brought as consequence an impact on the environment of the communities. The appearance of popular consultations that look for the opinion of the citizens with the purpose of preventing the accomplishment of the above mentioned activities has provoked a discussion on the autonomy of the territorial entities with regard to these topics. In this article, it is stated how the condition of the mining activity in the country is, furthermore, there is a checked of the legal frame of the popular consultations, the developed ones in mining topics and the legal response of the National Government that seeks to preserve the autonomy of the managing of the mining classification. The legal decisions adopted to the date are mentioned, and so the conclusions in which there is a real and actual Social State of Law.
\end{abstract}

Key Words: Democracy, Mining industry, Popular Consultation, Territorial autonomy

\section{Introducción}

El impulso que las actividades mineras han tenido en Colombia durante los últimos años han dinamizado la economía e impulsado al Gobierno Nacional a adoptar medidas que permitan atraer inversiones que promuevan la explore y exploten los recursos con los que cuenta el país. Esta clase de actividades genera roces con los ciudadanos que velan por el respeto al medio ambiente y que desde la Constitución de 1991 encuentran un respaldo en la carta constitucional para hacer respetar sus derechos. En el presente artículo se realizara en su primer aparte una revisión al estado de la actividad minera en el país. En el segundo aparte se realizara una descripción del proyecto de La Colosa ubicado en el Departamento del Tolima que por su importancia y magnitud está en la lupa de la comunidad. El tercer aparte realiza una revisión de las normas legales que regulan la consulta popular, uno de los mecanismos de participación ciudadana, establecido en la Constitución. El cuarto aparte aborda el asunto de las Consultas populares llevadas a cabo en Piedras (Tolima) y Tauramena (Casanare) y el ejercicio fallido de Monterrey (Casanare) En el quinto aparte se presenta la posición del gobierno nacional y los mecanismos legales utilizados para conservar el monopolio absoluto del ordenamiento minero. En las conclusiones se recogen los temas abordados y se indica que la discusión que subyace a estos temas más que interés económicos lo que confronta es en realidad la clase de Estado que rige actualmente en Colombia.

\section{La actividad minera en Colombia}

La minería no ha sido una actividad económica desconocida en Colombia. Al contrario, desde las épocas precolombinas donde las culturas indígenas realizaban valorados trabajos de orfebrería, la actividad ha estado presente en la vida del país. En la época colonial la minería creció, especialmente impulsada por los comerciantes antioqueños que transportaban el oro en polvo a otras regiones del país e incluso del exterior con la finalidad de intercambiarlas por textiles y alimentos. En el periodo de la República, la actividad minera se centró en el oro y las piedras preciosas, productos que fueron exportados y permitieron el equilibrio de la balanza comercial durante gran parte del siglo XIX, el siglo XX marca el inició de la explotación de otros metales. La llegada de las primeras máquinas de vapor a finales de la primera década del siglo permite el inicio de la explotación del carbón en pequeñas cantidades. En la década de 1950 se comienza a observar la integración vertical del carbón como insumo para la producción de papel y cemento. En la década de los ochenta, con el hallazgo de un yacimiento de ferroníquel en el departamento de Córdoba y el inicio de actividades de explotaciones carboníferas de gran escala en los departamentos de César y la Guajira el sector comienza a tener una importancia estratégica en la economía colombiana.(Cárdenas y Reina, 2008)

Debido al incremento del precio de los minerales ocurridos en la última década del siglo XX, se presenta un aumento de los ingresos del país por ese 
concepto. A inicios del Siglo XX la participación del sector minero en el PIB era de 1,8\%, y doce años después era del 2,4\%.(Villar, 2013). Adicionalmente, con la llegada del presidente Alvaro Uribe al poder se impulsó de manera importante la actividad minera. Su gobierno entregó en concesiones mineras entre 2004 y 2008 el equivalente al 10\% del territorio colombiano. (Velez, 2010). Entre julio y octubre de 2009 se concedieron o renovaron 1900 licencias para explotaciones mineras en los páramos.(Osorio, 2010). El Presidente Santos que fue elegido para el mandato 2010-2014 tuvo entre sus políticas de gobierno más importantes para su primer cuatrenio el impulso de la denominada "locomotora minera" que busca el impulso de la minería en el país, para lo cual se busca atraer inversión foránea que realice actividades de exploración y explotación. El sector minero unido al energético atrajo el $60 \%$ de la inversión extranjera directa en 2011 y para el año 2012 representaba el $66 \%$ del total de las exportaciones colombianas. (Mesa, 2013)

Los títulos mineros son los actos administrativos otorgados a personas naturales o jurídicas mediante los cuales el Estado autoriza a un particular a realizar actividades de exploración y explotación en el territorio colombiano. Es decir, para poder ejercer de manera legal estas actividades, debe tenerse previamente este acto administrativo mediante el cual se delimita el lugar y extensión del área donde se realizaran actividades mineras. Los requisitos que deben cumplirse para acceder a tal titulación no revisten ninguna dificultad. La única limitación que impide el otorgamiento de tal documento es que el área en que se solicita la titulación sea prohibida la realización de estas actividades por normas nacionales o municipales o la misma se encuentre dentro del territorio de resguardos indígenas o grupos étnicos, en cuyo caso es necesaria la autorización de tales grupos. (Ministerio de Minas, 2009)

Hasta el año 2012 la entidad encargada de expedir los títulos mineros era el Instituto Colombiano de Ingenieria y Minas (Ingeominas), institución ante la cual los particulares radicaban la documentación solicitada y obtenían el correspondiente título minero. Las graves denuncias realizadas sobre la "feria" de títulos realizadas en la entidad entre 2006 y 2010 (Revista Semana, 2011) fue una de las causas que motivaron que se creara la Agencia Nacional de Minería con la finalidad que ésta importante función se manejara de manera transparente y profesional por esta nueva entidad.
Lo descrito anteriormente implico la expedición de actos administrativos por parte del Estado en favor de particulares para la realización de actividades mineras aún en zonas de protección, situación que ha dado como resultado una disputa entre los derechos adquiridos de los favorecidos que deben respetarse y las normas ambientales que impiden la ejecución de actividades de exploración y explotación minera en suelos de protección

De la misma manera, existen zonas del país que fueron objeto de titulación sin el conocimiento de los habitantes del sector donde se realizara la exploración y/o explotación de recursos naturales. En síntesis, en los últimos quince años se observa un importante crecimiento de la actividad minera en Colombia fortalecida por las políticas pública de los últimos dos gobiernos que se la jugaron por dicho sector. Las debilidades institucionales de la entidad encargada de regular la actividad permitieron una explosión de títulos que otorga derechos a sus beneficiarios, quienes pretenden hacerlos valer aún contra normas ambientales existentes en el ordenamiento colombiano. Los ciudadanos de algunas de las zonas donde se realizaran este tipo de emprendimientos mineros se oponen la realización de las mismas por diversos motivos, uno de ellos es considerar que deben ser consultados de manera previa a la intervención del territorio con la finalidad de autorizar cualquier actividad en el mismo. El Gobierno que por mandato constitucional es propietario del subsuelo considera que tiene completa potestad para otorgar licencias a particulares con la finalidad de lograr la explotación de los recursos naturales. La manera en que se manejen los conflictos que se generan entre el Estado y los emprendedores mineros de una parte y los ciudadanos que viven en los territorios que se constituyen en área de influencia de un proyecto y se oponen a éste de otra debe revisarse para evitar que dichas diferencias terminen tramitándose de manera violenta y por fuera de los espacios del Estado de Derecho.

\section{El proyecto de explotación de Oro de La Colosa}

La empresa multinacional Anglo Gold Ashanti (en adelante AGA) con sede en Suráfrica es la tercera productora mundial de oro. En el año 2003 inició actividades en Colombia y en el año 2006 realizo el descubrimiento de un yacimiento de oro a 14 kilómetros del casco urbano del municipio de Cajamarca y 30 kilómetros al oeste de Ibagué, capital del departamento del Tolima. (Documento AGA, 2011) 
Las primeras noticias del descubrimiento del yacimiento de oro indicaban unas reservas de 12,9 millones de onzas, que fueron incrementándose al doble y de conformidad con lo expresado en el estudio de Colombia Solidarity denominado " $\mathrm{La} \mathrm{Co}$ losa : Una muerte anunciada." (2013), en el informe a los accionistas de AGA de 2012 las reservas fueron tasadas en 35 millones de onzas, que implicaría una suma superior a los 60.000 millones de dólares. Según el mismo informe mencionado, los principales interrogantes con relación al proyecto pueden resumirse de la siguiente manera. La explotación del material se realiza mediante explosiones y maquinaria. El material que surge del anterior ejercicio se divide en el que contiene el metal y aquel que no lo contiene. Este último tiene que ser depositado en escombreras de gran magnitud. Las rocas que contienen el oro son a su vez trituradas y molidas. Una vez realizado el anterior proceso, en una planta industrial se lleva a cabo el proceso de lixiviación con cianuro para extraer el oro para su posterior fundición. El anterior procedimiento genera unos residuos tóxicos que deben ser almacenados. Para esta actividad se crea una especie de represa y los residuos son llevados allí mediante unas estructuras que se denominan diques de cola. El anterior procedimiento exige especial cautela con relación al volumen de los escombros y el lugar donde estos se alojarían debido a que éste tipo de elementos pueden generar efectos negativos sobre los terrenos y el agua. Igualmente, el lugar donde se depositarían los residuos finales que vendrían transportados en el dique de colas y que supone un área diferente a la de explotación debido a las condiciones topográficas de Cajamarca. La construcción de un dique de 250 metros de altura con capacidad superior a 1400 millones de toneladas aumentaría de manera importante el riesgo de los terrenos aledaños a la zona de influencia del proyecto.(BM Colombia Solidarity, 2013)

Anunciado el descubrimiento a finales de 2007, durante el año siguiente fue detenida la etapa de exploración debido a que la Corporación Autónoma Regional del Tolima - Cortolima-, ${ }^{1}$ determinó

1 El artículo 23 de la ley 99 de 1993 dispone: Las Corporaciones Autónomas Regionales son entes corporativos de carácter público, creados por la ley, integrado por las entidades territoriales que por sus características constituyen geográficamente un mismo ecosistema o conforman una unidad geopolítica, biogeográfica o hidrogeográfica, dotados de autonomía administrativa y financiera, patrimonio propio y personería jurídica, encargados por la ley de administrar, dentro del área de su jurisdicción, el medio ambiente y los recursos naturales renovables y propender por su desarrollo sostenible, de conformidad con las disposiciones legales y las políticas del Ministerio del Medio Ambiente. que se estaban realizando exploraciones en zonas de reserva forestal. Seguido a lo anterior, en 2009 el Ministerio de Ambiente inicia una investigación contra AGA pero en mayo del mismo año dispone que 6,39 Has se extraen de la reserva con la finalidad que la empresa pueda realizar actividades de exploración en dicha área. No obstante lo anterior, en 2010 el Ministerio multa a AGA con $\$ 140.000 .000$ por haber realizado actividades de exploración sin haber solicitado antes la extracción del área de la zona de reserva. En 2011 la Contraloría General de la Nación denuncia que las exploraciones superan el área extraída de la reserva. Finalmente en 2012 se solicita la ampliación del área de extracción en 1,16 Has, la cual es autorizada por el Ministerio en mayo de 2013. Paralelo a lo anterior se intensifican las actividades de adquisición de predios en el área de influencia del proyecto. (BM Colombia Solidarity, 2013)

El municipio de Cajamarca donde se encuentra ubicado el yacimiento de Oro fue fundado en 1913, tiene una extensión total de 513 kilómetros cuadrados, de los cuales 354 kilómetros pertenecen a su área rural. Su altura sobre el nivel del mar es de 1814 metros y es conocido como "la despensa agrícola de Colombia" debido a la gran cantidad de productos agrícolas que se cultivan en sus suelos. Su población es de 19.789 habitantes. (Alcaldía de Cajamarca). De otra parte, el municipio de Piedras que fue fundado en 1552 tiene una extensión de 355,15 kilómetros cuadrados, de los cuales 354,56 corresponden al área rural. Se encuentra ubicado a 50 kilómetros de Ibagué, capital del Departamento del Tolima y su altitud es de 403 metros sobre el nivel del mar (Alcaldía de Piedras) Según el Censo de 2005, su población es de 5.370 habitantes. (Dane, 2005)

Es claro que el yacimiento de oro se encuentra dentro del territorio del municipio de Cajamarca y allí se realizaría la extracción del mismo. En el municipio de Piedras se evalúa la posibilidad de establecer el lugar para el tratamiento y almacenamiento de las enormes cantidades de material provenientes del tajo de la mina. AGA inició estudios de suelos en algunos municipios aledaños a Cajamarca y el 5 de diciembre de 2012 en una reunión en el Corregimiento de Doima del municipio de Piedras informaron a la comunidad de las labores que adelantaban. (BM Colombia Solidarity, 2013). Para el momento de realización de la citada reunión, la comunidad ya tenía un concepto negativo sobre el proyecto minero, del cual conocieron su existencia por los medios de comunicación y no por funcionarios del Gobier- 
no Nacional o la AGA. Previo a la mencionada reunión, activistas del medio ambiente y líderes comunitarios habían iniciado un proceso de concientización sobre los efectos que un proyecto del tamaño de la Colosa puede tener sobre la región y sus habitantes. En junio de 2011 se realizó en Ibagué y varios municipios del Tolima el carnaval en defensa del agua, la vida, el territorio y la soberanía alimentaria voluntaria donde participaron 12.000 personas. Este evento se reiteró en 2011, 2012 y en su quinta versión en 2013 reunió más de 30.000 personas, contando con la participación de parte importante de la dirigencia regional y local. Los Comités Ambientales y Campesinos del Tolima auspiciaron movimientos sociales que cerraron filas en contra del proyecto minero, situación que se concretó en episodios como el denominado "Planton" realizado en enero de 2013 cuando los habitantes del Corregimiento de Doima impidieron el acceso de los camiones y remesas de AGA al campamento que tienen en dicho lugar. A partir de allí, la comunidad ha mantenido su posición de impedir el trabajo de equipos y personas de la multinacional en su territorio (Corredor Gil, 2014).

AGA realizó estudios en el departamento del Tolima que se hicieron públicos hasta finales del año 2007 con el hallazgo de La Colosa. A partir de allí, realizaron algún contacto con la comunidad del municipio de Cajamarca y continuaron realizando actividades relativas al proyecto en otros municipios, entre los cuales se encontraba Piedras con la finalidad de buscar escenarios donde pudiera depositarse el importante número de escombros que dejara la explotación de oro. Su demora en informar a la comunidad y hacerla participe del proceso tuvo como consecuencia que la misma sintió que no fue tenida en cuenta en el proceso y cuando la empresa trato de recuperar el espacio perdido a causa de su propia omisión encontró una ciudadanía informada con una antipatía documentada hacia el proyecto minero que busca ser escuchada y que busca escenarios para que su opinión tenga eco. Una de las herramientas que es usada por los opositores a la explotación de oro es la consulta popular, la cual se mencionara a continuación.

\section{La Consulta Popular como mecanismo de par- ticipación ciudadana en la Constitución Política de Colombia}

Una de los pilares de la Constitución Política de Colombia es la participación de los ciudadanos que garantice un orden económico social y justo, tal como se menciona en el preámbulo de la carta.
De los artículos 103 a 105 del texto constitucional se establecen las formas de participación democrática. El artículo 103 indica:

Son mecanismos de participación del pueblo en ejercicio de su soberanía: el voto, el plebiscito, el referendo, la consulta popular, el cabildo abierto, la iniciativa legislativa y la revocatoria del mandato. La ley los reglamentará.

La ley 134 de 1994 se encargó de reglamentar los mecanismos de participación ciudadana mencionados en la carta política. En los artículos 50 a 57 se regula todo lo relacionado con la Consulta Popular. Este mecanismo de participación consiste en la consulta que realiza a la ciudadanía el Presidente de la República, el Gobernador de un Departamento o el Alcalde Municipal sobre un asunto de trascendencia nacional, departamental o local respectivamente. El mecanismo es el siguiente: El ejecutivo elabora la pregunta que se le va consultar a la ciudadanía que solo puede tener como respuesta sí o no. Esa iniciativa es presentada al Senado si es Nacional, la Asamblea en el caso de los Departamentos y el Concejo en el caso de los municipios con la finalidad que estos organismos aprueben la conveniencia de realizar la consulta. Una vez recibida esta aprobación y para las consultas departamentales y locales, se realiza un examen de la pregunta por parte del Tribunal Administrativo para que informe si la pregunta a realizar se encuentra acorde a la Constitución Política. Una vez el Senado aprueba la consulta, la misma se realizara dentro de los cuatro meses siguientes, en el caso de las consultas departamentales y locales se realiza dentro de los dos meses siguientes. En el caso de las consultas departamentales y locales, el texto de la consulta deberá remitirse al tribunal de lo contencioso administrativo competente con la finalidad que examine la constitucionalidad de la pregunta realizada. En la eventualidad que el juez administrativo la considere contraria a la Carta polítca no será viable la realización de la misma. Para que sea aprobada la consulta por parte de la ciudadanía se requiere el voto afirmativo de la mitad más uno de los sufragios y que hubiere participado la tercera parte de los electores que conforman el censo electoral. El artículo 56 dispone sobre los efectos de la consulta:

...Cuando el pueblo haya adoptado una decisión obligatoria, el órgano correspondiente deberá adoptar las medidas para hacerla efectiva. Cuando para ello se requiera de una ley, una ordenanza, un acuerdo o una 
resolución local, la corporación respectiva deberá expedirla dentro del mismo periodo de sesiones y a más tardar en el período siguiente. Si vencido este plazo el Congreso, la asamblea, el Concejo o la junta administradora local, no la expidieren, el Presidente de la República, el gobernador, el alcalde, o el funcionario respectivo, dentro de los tres meses siguientes la adoptará mediante decreto con fuerza de ley, ordenanza, acuerdo o resolución local, según el caso. En este caso el plazo para hacer efectiva la decisión popular será de tres meses."

De la reglamentación de la consulta popular se puede inferir que: a-) Su iniciativa corresponde al ejecutivo nacional, departamental o local. b-) Para el evento de las consultas departamentales y locales se requiere la validación de la misma por las Asambleas y Concejos y la revisión previa de un tribunal de la jurisdicción contencioso administrativa. C-) Existe un control judicial previo de constitucionalidad realizado por los tribunales administrativos que busca salvaguardar los derechos consagrados en la carta política d-) Es condición indispensable para la validez de la consulta que participe en la misma una tercera parte del censo electoral nacional, departamental o local según sea el caso. e-) La aprobación de la consulta por parte del pueblo tiene como consecuencia para el gobierno nacional, departamental o local, la obligatoriedad de adoptar las medidas para hacer efectiva la decisión consultada.

La primera consulta popular realizada en Colombia data de agosto 27 de 1995 en el Municipio de Aguachica ubicado en el departamento del César en el cual se le pregunto a los habitantes de este municipio: "¿Rechaza usted la violencia y está de acuerdo en convertir a Aguachica en un municipio modelo de paz?". En el municipio se vivían un especial momento de tensión social por las acciones violentas que se adelantaban en su territorio por diversos actores. Con la finalidad de unir a la comunidad para rechazar esta situación e impulsar un modelo distinto de convivencia el Alcalde Municipal lidero la consulta en la que participaron 10.569 personas con voto afirmativo superior al 99,5\%, sin embargo debido a que el censo electoral era de 34.505 ciudadanos, no se alcanzó la participación mínima requerida por la ley y por ello dicha consulta no procedió. Sin embargo, esta situación dirigió el foco del país a ese municipio y se lograron atención e inversiones nacionales y departamentales con posterioridad la realización de la consulta. (López y Navarro, 2005)
Desde la vigencia de la ley 134 que comenzó a regir el 31 de mayo de 1994 y hasta el primer semestre del año 2014 se han realizado 33 consultas populares según la información que brinda la Registraduria Nacional del Estado Civil, entidad que tiene la función de organizar y vigilar los procesos electorales en Colombia. De las consultas realizadas se pueden realizar las siguientes anotaciones:

a-)Todas las consultas han sido municipales. Este mecanismo de participación ciudadano no ha sido estrenado por presidentes ni gobernadores de departamento.

b-) Los temas que han sido sometidos a consulta a los ciudadanos se han referido a los siguientes aspectos: - Cuatro consultas sobre integración de áreas metropolitanas. ${ }^{2}$

- $\quad$ Cinco Consultas relacionadas con temas de paz. ${ }^{3}$

- Diez consultas sobre aspectos de integridad territorial referentes a la anexión de municipios y creación de los mismos.

- Doce Consultas sobre aspectos puntuales de gobierno desde la adquisición de lotes para fines específicos, la prohibición de juegos de azar, hasta la construcción de plazas municipales, entre otros temas.

- Con relación a temas de prohibición de actividades de exploración y explotación de recursos naturales se han realizado dos consultas populares en los municipios de Piedras (Tolima) y Tauramena (Casanare) en el segundo semestre de 2013. Una tercera consulta sobre la misma temática que había sido programada para abril de 2014 en el municipio de Monterrey en el departamento del Casanare en abril de 2014 fue suspendida por el Consejo de Estado.

2 El artículo 2 de la ley 1625 de 2013, con relación a las áreas metropolitanas dispone que: "Las Áreas Metropolitanas son entidades administrativas de derecho público, formadas por un conjunto de dos o más municipios integrados alrededor de un municipio núcleo, vinculados entre sí por dinámicas e interrelaciones territoriales, ambientales, económicas, sociales, demográficas, culturales y tecnológicas que para la programación y coordinación de su desarrollo sustentable, desarrollo humano, ordenamiento territorial y racional prestación de servicios públicos requieren una administración coordinada." 3 Adicionalmente a la Consulta realizada en Aguachica, se realizaron consultas en Santa Marta capital del departamento del Magdalena en 1995 con una pregunta igual y tres consultas en diciembre 19 de 1999 en los municipios guajiros de Fonseca, SanJjuan del César y Villanueva con la pregunta: "Rechaza usted que sigan involucrando a la población civil en la guerra, y está de acuerdo en hacer de San Juan del Cesar un modelo de convivencia pacífica?" 
4. La Consulta Popular de Piedras y sus consecuencias. Validez de la prohibición ciudadana de adelantar actividades mineras en su territorio.

El Alcalde del Municipio de Piedras en el Departamento del Tolima puso a consideración del Concejo Municipal el texto de la consulta popular que sobre temas relativos a las actividades mineras pretendía realizar a los habitantes del municipio. El Concejo Municipal, el 18 de mayo de 2013 dio un concepto favorable a la realización de la citada consulta. El texto de la pregunta fue enviado al Tribunal Administrativo del Tolima con la finalidad que examinara la constitucionalidad de la misma. En Sentencia de Junio 26 de 2013, el Tribunal encontró ajustada a la Constitución el procedimiento realizado y dio vía libre al mecanismo de participación ciudadana. En dicha providencia destacó esta instancia judicial que:

\section{...Revisado su contenido y sustento en los fundamentos legales y jurisprudenciales ex- plicados en esta providencia, tenemos que la pregunta formulada y esgrimida en líneas previas, se encuentra proyectada de mane- ra adecuada, dando claridad a la población de que su decisión cualquiera sea su rigor, será significativa, ya que son ellos los direc- tamente interesados en el tema para el bien- estar de su municipio.}

Es evidente que la Constitución privilegia el derecho al medio ambiente y conservación de un ambiente sano a través de los artículos $1^{\circ}, 58,80$ y 95, entre otros. Véase lo que al respecto ha dicho la Corte Constitucional:

...Una teórica discusión jurídica en materia ambiental, sobre cuáles derechos prevalecen, la resuelve la propia constitución, al reconocer la primacía del interés general, bajo las condiciones del artículo $1^{\circ}$. Al señalar que la propiedad privada no es un derecho absoluto, sino que "es una función social que implica obligaciones. Como tal, le es inherente una función ecológica (art. 58, inc. $2^{\circ}$ ).

Además, señala la Constitución, que el Estado debe

"prevenir y controlar los factores de deterioro ambiental, imponer las sanciones legales y exigir la reparación de los daños causados" (art. 80). Así mismo, establece dentro de los deberes de la persona y el ciudadano la obligación de "proteger los recursos culturales y naturales del país y velar por la conservación de un ambiente sano (art. 95 ord. $8^{\circ}$ )

Mediante decreto 095 del 9 de julio de 2013 el Alcalde de Piedras, municipio del departamento del Tolima, convocó una consulta popular para responder la siguiente pregunta:

...Está de acuerdo, como habitante del municipio de Piedras Tolima que se realice en nuestra jurisdicción, actividades de exploración, explotación, tratamiento, transformación, transporte, lavado de materiales provenientes de las actividades de explotación minera aurífera a gran escala, almacenamiento y el empleo de materiales nocivos para la salud y el medio ambiente de manera específica el cianuro y / o cualquier otra sustancia o material peligroso asociado a dichas actividades y se utilicen las aguas superficiales y subterráneas de nuestro municipio en dichos desarrollos o en cualquier otro de naturaleza similar que pueda afectar y / 0 limitar el abastecimiento de agua potable para el consumo humano, la vocación productiva tradicional y agrícola de nuestro municipio.

Con un censo electoral de 5.105 personas, participaron en la consulta 3.007 personas, de las cuales 2.971 votaron por él No, 24 votaron por él sí, hubo 2 votos nulos y 10 tarjetas no marcadas. (Fuente, Registraduría Nacional del Estado Civil).

La Consulta adelantada en el municipio de Piedras cumplió con todos los requisitos exigidos por la ley para su validez y efectividad. Con la realización de esta consulta se dio inicio a un debate sobre la competencia de los municipios para regular aspectos relacionados con la actividad minera. La posición del gobierno nacional y la industria minera es que la competencia para regular todos los aspectos del proceso minero radica única y exclusivamente en el ejecutivo nacional. Previo a abordar esta discusión, conviene conocer los aspectos de otras dos consultas que sobre temas similares se tramitaron en dos municipios del departamento del Casanare. Luego de la experiencia de Piedras, los municipios de Tauramena y Monterrey comenzaron el trámite para la realización de sus consultas populares que buscaban preguntarle a la población su opinión sobre la posibilidad de que en sus municipios se iniciaran actividades de exploración sísmica en cercanía a las zonas de recarga hídrica. En Tauramena, luego de cumplir los procedimientos legales, la consulta se realizó el 15 de diciembre de 2013 con la participación de 4.577 personas de 
un censo electoral de 13.372. (Registraduria Nacional del Estado Civil, 2013)

La pregunta realizada a los ciudadanos de Tauramena fue la siguiente:

...Está usted de acuerdo con que se ejecuten actividades de exploración sísmica, perforación exploratoria, producción y transporte de hidrocarburos, en las veredas San José, Monserrate Alto, Monserrate La Vega, Guafal del Caja, Bendiciones, Visinaca, Lagunitas, Aguamaco, Zambo, Oso y Jaguito, donde se ubica la zona de recarga hídrica del municipio de Tauramena?

4.426 ciudadanos votaron negativamente mientras que 151 votaron de manera positiva.. Participo más de la tercera parte del censo electoral del municipio.

En el municipio de Monterrey en el mes de noviembre de 2013 se iniciaron los trámites de una consulta popular con una pregunta similar a la de Tauramena. El Concejo Municipal dio vía libre a la iniciativa y el 13 de diciembre se radico en el Tribunal Administrativo de Casanare para el examen de constitucionalidad exigido por la ley. En desarrollo de dicho examen, el Tribunal organizo de manera facultativa una audiencia para escuchar las opiniones de las diferentes autoridades de la región sobre la consulta propuesta. La Empresa Colombiana de Petroleos -Ecopetrol- interpuso una acción de tutela contra la autoridad judicial indicando que se violaba su derecho fundamental al debido proceso al no ser escuchada en la audiencia citada por el Tribunal. Las peticiones de la Empresa tuvieron acogida en el Consejo de Estado y se tutelaron sus derecho al debido proceso, en los cuales se indicó que al no estar comprendida en la normatividad la audiencia que pretendía realizar el Tribunal, se estaba vulnerando normas de la carta política. En razón a ello el Tribunal el 7 de febrero de 2014 prescindió de la audiencia y en decisión de febrero 27 de 2014 encontró ajustado a la Constitución el procedimiento adelantado y la pregunta a realizarse. La consulta fue programada para el 27 de abril de 2014.

La pregunta que se realizaría a los ciudadanos era:

...iEstá usted de acuerdo con que se ejecuten actividades de exploración sísmica, perforación exploratoria, producción y transporte de hi- drocarburos en las veredas Cacical, Guayabal, Piñalera y Guadualito, donde se ubica la zona de recarga hídrica del municipio de Monterrey?

No obstante lo anterior, tres días antes de la realización de la Consulta, el Consejo de Estado al admitir una acción de tutela contra la decisión del Tribunal Administrativo del Casanare ordeno la suspensión de la misma como medida cautelar.

Ecopetrol considero que se violaban sus derechos al debido proceso, al trabajo y le ocasionaban un perjuicio directo debido a que el área objeto de la consulta debía cumplir obligaciones por un valor superior a los 57.000 millones de pesos. Se argumentó también que la decisión que pretendía ponerse a consideración de los habitantes del municipio excedía las competencias municipales. En el fallo de fondo, el Consejo de Estado tutela los derechos de Ecopetrol considerando que la consulta no solo buscaba proteger las fuentes hídricas sino restringir la realización de actividades de la empresa tutelante que había adquirido compromisos previos a la formulación de la iniciativa.

\section{La "competencia exclusiva" del Gobierno Na- cional sobre asuntos mineros}

Debido a la importancia que ha venido adquiriendo para el país en los últimos años todo lo relevante al tema minero energético como se mencionaba en el primer acápite del presente artículo ante la posibilidad que en diferentes lugares del país movimientos ciudadanos impulsaran consultas populares similares a las mencionadas, el Gobierno Nacional resolvió tomar medidas en el asunto y para ello expidió el Decreto 934 de mayo 9 de 2013 mediante el cual reglamentaba el artículo 37 de la ley 685 de 2001.

El artículo 37 de la citada Ley, que es el Código de Minas, dispone:

Con excepción de las facultades de las autoridades nacionales y regionales que se señalan en los artículos 34 y 35 anteriores, ninguna autoridad regional, seccional o local podrá establecer zonas del territorio que queden permanente o transitoriamente excluidas de la minería. Esta prohibición comprende los planes de ordenamiento territorial de que trata el siguiente artículo.

El Decreto 934 de 2013 reglamenta el anterior artículo, indicando entre otras cosas: 
a-) Que las únicas autoridades ambientales que pueden establecer zonas restringidas y excluidas de minería son: El Ministerio de Ambientes y Desarrollo Sostenible y Las Corporaciones Autónomas Regionales. La autoridad minera es la Agencia Nacional de Minería o quien haga sus veces.

b-) Los planes de ordenamiento territorial no pueden contener ninguna disposición relativa a ordenamiento minero salvo que hubiere sido aprobada por las anteriores autoridades.

c-) Los Concejos municipales o las asambleas departamentales no pueden expedir acuerdos ni ordenanzas que excluyan temporal o permanente un área de la actividad minera por carecer de competencia y en la eventualidad que en los instrumentos de ordenamientos quedaran consignadas dichas limitaciones, las mismas no serán oponibles ni aplicadas por ninguna autoridad. Por lo anterior los certificados de uso de suelo municipales que informen de tales afectaciones no podrán ser tenidos en cuenta por las autoridades que tramitan licencias o permisos para el ejercicio de la actividad minera.

En síntesis: La competencia para establecer áreas donde se limita parcial o totalmente la actividad minera recae de manera exclusiva en el Gobierno Nacional. Como consecuencia de lo anterior, una consulta popular que disponga algo relacionado con el ordenamiento minero y que sea elevado a norma municipal o departamental no tiene ninguna clase de efecto sobre esta actividad económica.

La anterior tesis que parece sólida ha comenzado a tener fisuras debido a demandas de inconstitucionalidad presentadas ante la Corte Constitucional y el Consejo de Estado. Ante el máximo tribunal constitucional fue presentada una demanda de inconstitucionalidad contra el artículo 37 de la ley 685 de 2001. La ciudadana Zulma Tatiana Blanco demando la norma aludida indicando que la misma viola el principio de autonomía territorial y auspicia el incumplimiento de la obligación del Estado de salvaguardar el patrimonio cultural y proteger el medio ambiente en Colombia. Mediante la Sentencia de Constitucionalidad 123 de marzo 5 de 2014 la Corte Constitucional declara la exequibilidad condicionada del artículo demandado indicando que

...en desarrollo del proceso por medio del cual se autorice la realización de actividades de exploración y explotación minera, las autoridades competentes del nivel nacional de- berán acordar con las autoridades territoriales concernidas, las medidas necesarias para la protección del ambiente sano, y en especial, de sus cuencas hídricas, el desarrollo económico, social, cultural de sus comunidades y la salubridad de la población, mediante la aplicación de los principios de coordinación, concurrencia y subsidiariedad previstos en el artículo 288 de la Constitución Política."

La Sentencia ha tenido dos lecturas: De una parte, el Gobierno y la industria minera han considerado que se confirma la competencia en cabeza del ejecutivo para el manejo de asuntos mineros. De otra parte, se considera que la Corte valido la necesidad de la concertación de las autoridades nacionales y locales, por lo cual éstas últimas deben ser tenidas en cuenta en la autorización de actividades minera. Otra lectura, es la que dan los magistrados Calle y Vargas en el salvamento de voto. Luego de expresar las razones por las cuales consideran que dicha norma es contraria a la Constitución, al vulnerar, entre otras cosas, la competencia de las autoridades territoriales en las decisiones relacionadas con la explotación de recursos naturales, la autonomía de los entes territoriales, las competencias para regular el uso del suelo que es una competencia asignada a los municipios y la participación de la comunidad en las decisiones que puedan afectar su entorno, los magistrados disidentes concuerdan en que la decisión adoptada se torna inocua al mencionar la concertación entre autoridades nacionales y locales pero no indicar la forma en que la misma deba realizarse. Esa ambigüedad ocasionara una participación formal de las autoridades locales en los asuntos relacionados con la actividad minero pero sin ningún efecto en la práctica.

La Contraloría General de la Nación presento en octubre de 2013 una demanda de nulidad por inconstitucionalidad ante el Consejo de Estado contra el Decreto 934 de 2013. La demanda se fundamenta principalmente en dos aspectos: El decreto no fue consultado con las comunidades étnicas siendo obligatoria su participación. Viola la autonomía de los municipios y distritos al impedir la reglamentación de los usos del suelo y la adopción de normas tendientes a la preservación y defensa del patrimonio ecológico. La demanda fue admitida en marzo de 2014 y se dio traslado a los interesados para que se pronuncien sobre la solicitud de suspensión provisional impetrada.

Tanto en el salvamento de voto comentado como en la demanda interpuesta por la Contraloría General de la República se esboza que la exclusión de 
los municipios en asuntos relacionados con el ordenamiento minero que se desarrollen en su territorio se convierte en una mutilación de sus funciones, un atropello a la descentralización y un desconocimiento a la participación de la comunidad en las decisiones que los afectan, valor fundamental en un Estado de Derecho. Despojar de influencia a los entes territoriales y las comunidades que en ellos habitan de las decisiones que puedan afectar el ambiente y su forma de vida con el argumento del interés nacional y de que la propiedad del subsuelo es del Estado es una decisión propia de un modelo de estado diferente al que actualmente rige el ordenamiento jurídico colombiano

\section{Conclusiones}

La importancia que la actividad minero energética tiene en Colombia está creciendo de manera acelerada.. Las políticas públicas que orientan estos temas desde hace tres lustros han buscado que la inversión extranjera se sientan atraída para movilizar capitales hacia la exploración y explotación de recursos naturales en el país. Con la finalidad de simplificar los trámites e impulsar esta actividad el Gobierno Nacional está resuelto a monopolizar el manejo de todo lo relacionado con el ordenamiento minero. Esta decisión que pudiera parecer conveniente desde el punto de vista gerencial tiene aspectos que contrarían el ordenamiento constitucional colombiano al despojar de funciones a entes territoriales.

Los grupos de ciudadanos que se oponen a las actividades mineras al considerarlas riesgosas para ciertas partes del territorio han movilizado a la comunidad para rechazar las mismas. En desarrollo de esa movilización se ha utilizado un mecanismo de participación ciudadana como la consulta popular que obligue a sus mandatarios a impedir la realización de actividades mineras. Comenzado en Piedras y siguiendo en Tauramena, las dos consultas populares adelantadas sobre el tema han tenido casi la unanimidad de los participantes en su oposición a la práctica de actividades mineras.
La respuesta del Gobierno ha sido expedir normas que confirman su posición de manejar de manera exclusiva el asunto. En el fondo, se vislumbra una confrontación entre dos posiciones: Los municipios que piden ser tenidos en cuenta en los procesos de desarrollo de la actividad minera y la comunidad que exige que su voz sea escuchada y en la eventualidad de repudiar la práctica de actividades mineras, su decisión sea respetada y el Gobierno Central que define el asunto minero como de interés nacional ante el cual no pude ni está dispuesto a compartir su poder de decisión. Pareciera que hasta el primer trimestre de 2014 la posición del gobierno ganaba de largo la discusión. Sin embargo, la decisión de la Corte Constitucional, que aunque bastante tímida índico que debe concertarse con los entes territoriales ha comenzado a iguala la balanza. La decisión que deberá adoptar el Consejo de Estado sobre la legalidad del Decreto 934 de 2103 será importante para conocer si las partes esta en plano de igualdad o la balanza se ha inclinado definitivamente a favor del Gobierno Nacional.

El resultado de estas disputas legales permitirá conocer si es viable en consonancia con la Constitución que los ciudadanos participen en las decisiones que los afectan sobre situaciones que ocurran en su territorio. Más que el tema minero lo que está en juego es la esencia del Estado Social de Derecho, pues si los ciudadanos no pueden adoptar decisiones sobre su entorno debido a los intereses económicos de la nación los valores y fundamentos de la carta política de 1991 habrían desaparecido. Supongamos que el área de los yacimientos de oro no afectara el municipio de Piedras sino el municipio de Anapoima, lugar de recreo de la élite capitalina. ¿Se aplicaría con el mismo rigor el interés nacional en el ordenamiento minero?

Las consultas populares que cumplan con los requisitos que establece la ley 134 de 1994 son de obligatorio cumplimiento para las autoridades. Menoscabar este mecanismo de participación ciudadana es regresar a un modelo de Estado que hoy no tiene Colombia 


\section{Referencias}

Alcaldía de Cajamarca, (2014) “Indicadores del Municipio". Recuperado el día 23 de Octubre de 2014 disponible en: http:/ / www.cajamarca-tolima.gov.co/indicadores.shtml\# otrosind

Alcaldía de Piedras, (2014) “Información General". Recuperado el día 20 de ocubre de 2014 disponible en: http: / / www.piedras-tolima.gov.co/informacion_general.shtml

Anglo Gold Ashanti, (2011),"Proyecto de exploración: La Colosa". Recuperado el día 23 de Octubre de 2014 disponible en: http: / / www.anglogoldashanti.com.co/nuestronegocio/Documentos/Proyecto_La_Colosa_20 11Dic.pdf:

BM Colombia Solidarity Campaign (2013), "La Colosa: Una muerte anunciada. Informe alternativo acerca del proyecto de Minería de Oro de Anglo Gold Ashanti en Cajamarca, Tolima, Colombia." Recuperado el día 25 de Octubre de 2014 disponible en: http: / / www.colombiasolidarity.org.uk/ attachments / article/610/LA\%20COLOSA_Una\%20Muerte\%20Anunciada.pdf

Cárdenas, M y Reina, M (2008), “La Minería en Colombia, Impacto Socioeconómico y Fiscal, Proyecto de la Cámara de Asomineros de la Asociación Nacional de Industriales",(2008) Recuperado el día 27 de Octubre de 2014 disponible en: http:/ / www.fedesarrollo.org.co/wp-content/ uploads / 2011/08/ La-miner\%C3\%ADa-en-Colombia-Informe-de-Fedesarrollo-2008.pdf

Corredor Gil, D (2014) “Estrategias de control ciudadano:Una aproximación a la intervención megaminera en Cajamarca Tolima. (2007-2013)", Tesis de grado de la Facultad de Ciencia Política y Gobierno de la Universidad Colegio Mayor de Nuestra Señora del Rosario. Recuperado el día 20 de septiembre de 2014 disponible en http://repository.urosario.edu.co/bitstream/handle /10336/5057/ 10101940592014.pdf?sequence=1

Corte Constitucional Colombiana, Sentencia C-123 de 2014 Magistrado Ponente: Alberto Rojas Ríos

Departamento Administrativo Nacional de Estadística (2005), “Censo general 2005.

López Silva M y Navarro Suárez C (2005), “Efectividad Socio Jurídica de la Consulta de Paz en Aguachica:", Tesis de Grado, Universidad Industrial de Santander, Recuperado el día 23 de Octubre de 2014 disponible en: http:/ / tangara.uis.edu.co/biblioweb / tesis / 2005/116768.pdf

Mesa Callejas, R (2013),"Aspectos macroeconómicos del auge minero energético en Colombia" en Seminario Interuniversitario de Economía, Regalías en Colombia y Estabilidad Macroeconómica, Universidad de Antioquia, Recuperado el día 17 de Octubre de 2014, disponible en: http:/ / www.udea.edu.co/portal/page/portal/bibliotecaSedesDependencias/unidadesAcademicas / FacultadCienciasEconomicas / ElementosDiseno / Documentos / Memorias / general / Aspectos\%20 macroecon\%C3\%B3micos\%20del\%20auge\%20minero-energ\%C3\%A9tico.pdf

Ministerio de Minas y Energia de Colombia (2009), Cartilla, Así es la Minería, Colombia. Recuperado el día 20 de agosto de 2014 Disponible en: http:/ / www.simco.gov.co/Portals/0/archivos/Cartilla_Mineria.pdf 